\title{
Stability of a Nonequilibrium Biochemical Cycle Revealed by Single-Molecule Spectroscopy
}

\author{
Saurabh Talele ${ }^{1,2}$ and John T. King ${ }^{1 *}$ \\ ${ }^{1}$ Center for Soft and Living Matter, Institute for Basic Science, Ulsan 44919, Republic of Korea \\ ${ }^{2}$ Department of Biomedical Engineering, Ulsan National Institute of Science and Technology, Ulsan 44919, \\ Republic of Korea
}

\begin{abstract}
Biological machinery relies on nonequilibrium dynamics to maintain stable directional fluxes through complex reaction cycles. In stabilizing the reaction cycle, the role of microscopic irreversibility of elementary transitions, and the accompanying entropy production, is of central interest. Here, we use multidimensional single-molecule spectroscopy to demonstrate that the reaction cycle of bacteriorhodopsin is coupled through both reversible and irreversible transitions, with directionality of trans-membrane $\mathrm{H}^{+}$ transport being ensured by the entropy production of irreversible transitions. We observe that thermal destabilization of the process is the result of diminishing thermodynamic driving force for irreversible transitions, leading to an exponentially increasing variance of flux through the transitions. We show that the thermal stability of the reaction cycle can be predicted from the Gibbs-Helmholtz relation.
\end{abstract}

Motor proteins operate through non-thermal motions (nonequilibrium fluctuations) [1-5] induced by the input of energy by, for example, ATP hydrolysis or photon absorption. Chemically driven motors have been adequately described using the principle of microscopic reversibility [6], where equilibrium mechanical motions of the protein are rectified by an asymmetric potential that biases diffusion in one direction [7-9]. Optically driven motors, in contrast, are thought to operate through a 'power-stroke' mechanism, where a series of conformational transitions follow from a sudden structural change $[3,10,11]$. For this physical mechanism, which is inherently far-from-equilibrium, the validity of equilibrium notions of time-reversal symmetry and detailed balance, and the stability of the reaction cycle to external perturbations, are yet to be established.

In this work, we study the reaction cycle of bacteriorhodopsin (bR), an optically-driven $\mathrm{H}^{+}$pump found in Archaea (Fig. 1a,b) [12], at the single-molecule level. Photon absorption induces a trans-cis isomerization of the retinal chromophore. Steric repulsions between the isomerized chromophore and the protein scaffold initiates a multi-step reaction cycle involving multiple intermediate species that exist for timescales ranging from $\mu$ s to ms during the cycle (Fig. 1c) $[13,14]$. Key to our understanding of nonequilibrium reaction cycles are the roles of time-reversal symmetry and the corresponding entropy production [15]. To characterize these properties on a single-molecule level, we leverage multidimensional single-molecule fluorescence lifetime correlation spectroscopy (sm-2D-FLCS) [16-19], which utilizes multiple light-matter interactions separated by a controlled waiting time to monitor structural or chemical transitions of a molecule. The exchange dynamics are observed through reciprocal time-correlation functions 

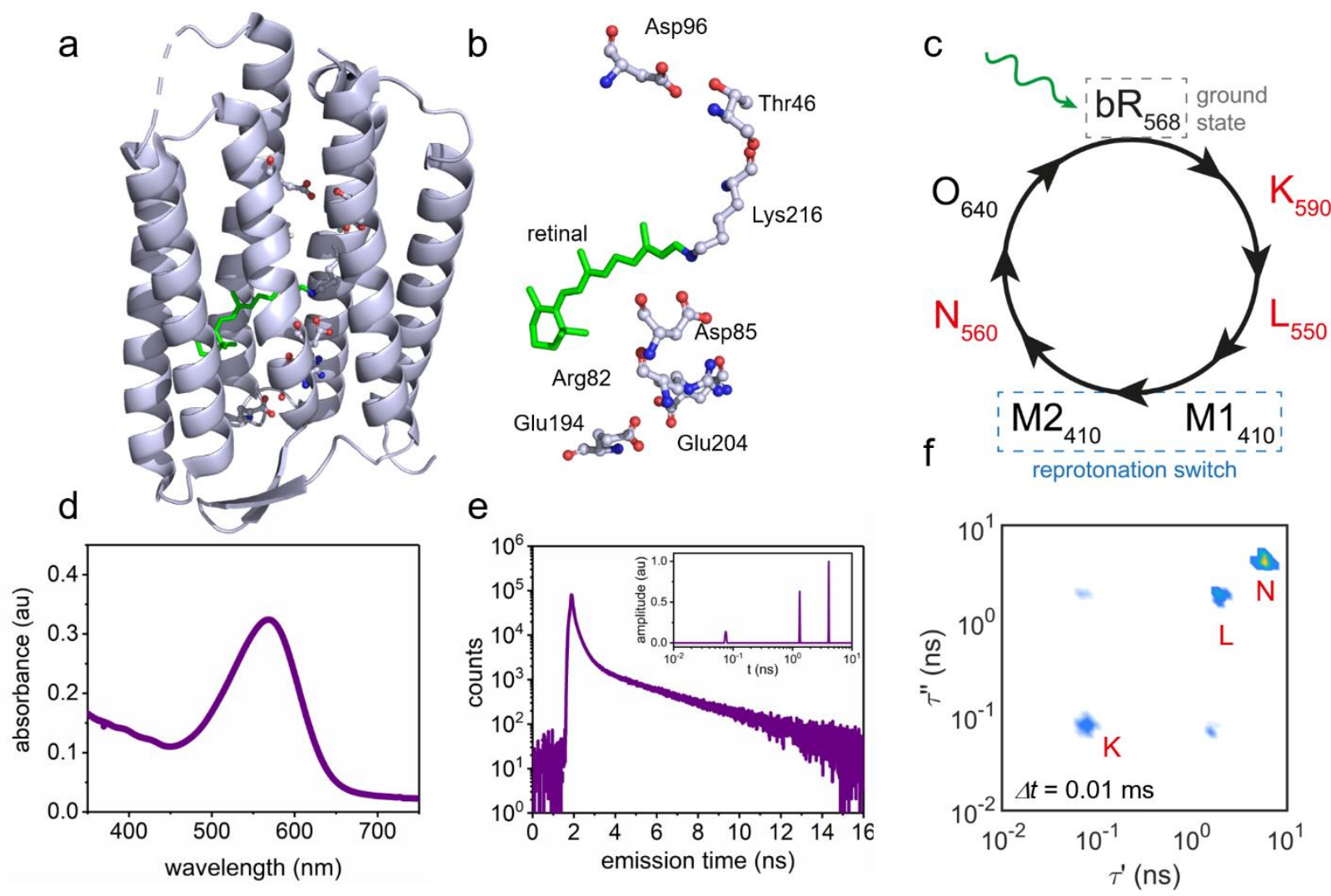

FIG. 1. Single-molecule spectroscopy of bR. Crystal structure of ground state bR showing (a) the protein (with the retinal chromophore shown in green) and (b) the retinal chromophore and the amino acids involved in the $\mathrm{H}^{+}$transport chain of the reaction cycle (PDB: $1 \mathrm{KBG).} \mathrm{(c)} \mathrm{Photo-}$ induced reaction cycle of $\mathrm{bR}$, which involves 6 intermediates in addition to the ground state (gs). The subscripts on the labels denote the absorption maximum of the retinal chromophore for the given conformation. (d) Absorption spectrum of retinal embedded in bR shows strong absorption at $\lambda=568 \mathrm{~nm}$ at $\mathrm{pH}=6$. (e) The fluorescence lifetime histogram measured from a single protein shows multi-component relaxation following $532 \mathrm{~nm}$ excitation. A 1D-ILT of the lifetime histogram reveals three relaxation times of $\tau_{1}{ }^{\prime} \sim 0.08 \mathrm{~ns}$ (K intermediate), $\tau_{2}{ }^{\prime} \sim 1.0 \mathrm{~ns}$ (L intermediate), and $\tau_{3}{ }^{\prime} \sim 5.0 \mathrm{~ns}$ ( $\mathrm{N}$ intermediate) (inset). (f) sm-2D-FLCS spectrum measured at a waiting time of $\Delta t=10 \mu \mathrm{s}$. Forward and reverse transition cross-peaks are already present between the $\mathrm{K}$ intermediate and the $\mathrm{L}$ intermediate are already observed at $10 \mu \mathrm{s}$, which indicates rapid exchange. In contrast, no cross-peaks are observed between the $\mathrm{N}$ intermediate and either the ground state or the L intermediate.

(tcf) for forward and reverse transitions, which appear in opposite quadrants of the 2D spectrum [20] and therefore allow the forward and reverse rates $\left(k_{f}\right.$ and $k_{r}$ ) can be measured independently. The violation of time-reversal symmetry within the reaction cycle is observed from the reciprocal tcf [21], which are equivalent for reversible transitions (obey time-reversal symmetry) and are not equivalent for irreversible transitions (violate time-reversal symmetry). The extent to which time-reversal symmetry is violated is a measure of entropy production rate in the cycle [15]. Using this experimental approach, we are able to experimentally characterize the nonequilibrium thermodynamics and kinetics of portions of the $b R$ reaction cycle, and quantify the stability of the cycle when subject to kinetic perturbation.

We first characterize the directional flux of the cycle by monitoring the transition kinetics between several intermediates. The endogenous retinal chromophore serves as the photo-trigger for the reaction cycle as well as a probe of the intermediates during the cycle. Limitations in the experiment prevent transitions between each 


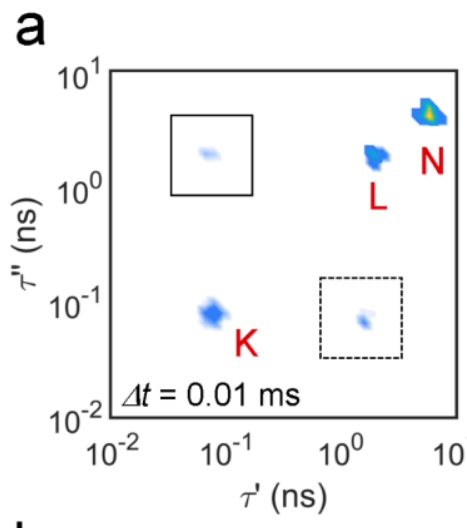

b

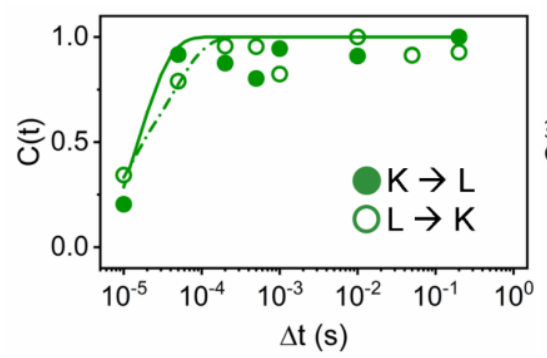

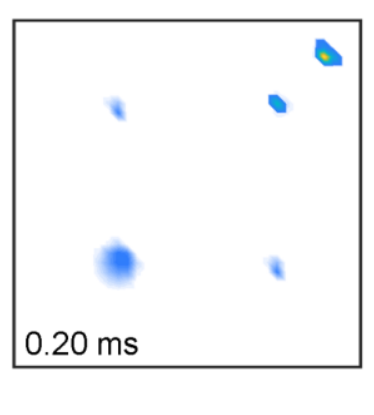
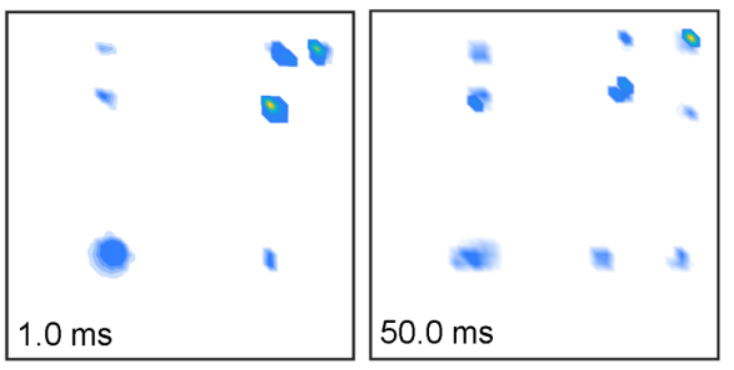

C

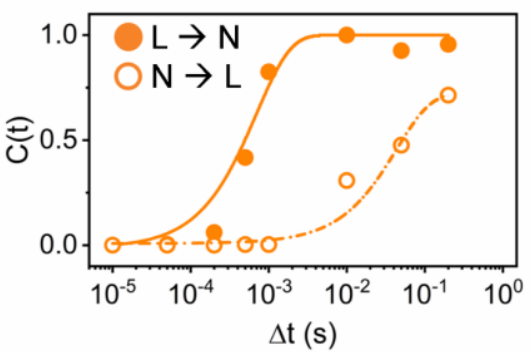

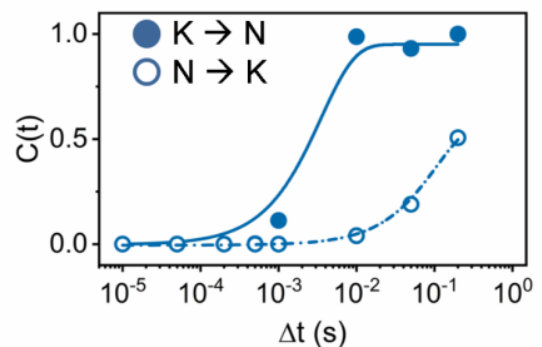

FIG. 2. Microscopic irreversibility of bR catalytic cycle. (a) sm-2D-FLCS spectra of bR shown for waiting times ranging from 0.01 to $50 \mathrm{~ms}$. Exchange kinetics for waiting times $\Delta t=0.01-200 \mathrm{~ms}$ between (b) $\mathrm{K}$ intermediate to $\mathrm{L}$ intermediate, (c) $\mathrm{L}$ intermediate to $\mathrm{N}$ intermediate, and (d) $\mathrm{K}$ intermediate to $\mathrm{N}$ intermediate. Forward transitions, represented by solid symbols and solid lines, are measured from the upper quadrant of the 2D spectra. Reverse transitions, represented by open symbols and dashed lines, are measured from the lower quadrant of the 2D spectra. The forward $\left(k_{f}\right)$ and reverse $\left(k_{r}\right)$ transition rates between the ground state and the L intermediate, measured by fitting the cross-correlation functions, are comparable, suggesting reversible dynamics and equilibration between these two states. In contrast, $k_{f}$ between the $\mathrm{L}$ intermediate and the $\mathrm{N}$ intermediate are two orders of magnitude larger than $k_{r}$, indicating a directional transition in the reaction cycle. The asymmetry in transition crosscorrelation functions (Eq. 1) indicates microscopic irreversibility of conformational transitions between the $\mathrm{L}$ and the $\mathrm{N}$ intermediates. This irreversible transition corresponds to the reprotonation switch step of the cycle.

intermediate to be directly measured. Instead, portions of the reaction cycle are characterized through select intermediates to which our experiments are sensitive. The cycle of bR involves six known intermediate structures that exist for timescales ranging between $\mu$ s to ms (K, L, M1, M2, N, O, Fig. 1c) [13, 14]. In the ground state structure, the retinal chromophore has an absorption maximum at $\lambda_{\max }=568 \mathrm{~nm}$ (Fig. 1d). Fluorescence emission from a single monomeric bR protein, confirmed by diffraction limited emission spots observed in confocal microscopy and singlestep photo-physics (Fig. S1a-c), shows multi-component relaxation following a $532 \mathrm{~nm}$ excitation (Fig. 1e, Fig. S1d). An inverse Laplace transform (ILT) [22] of the cumulative photon histogram measured from more than 100 single protein molecules (Fig. 1e) gives a 1D fluorescence lifetime spectrum that contains three distinct relaxation peaks (Fig. 1e, inset). We do not anticipate signal from the M1, M2, and O intermediates due to low absorption at the 532 $\mathrm{nm}$ excitation $[13,14]$. Furthermore, fluorescence from the ground state structure is unlikely due to the ultrafast isomerization reaction that occurs in the excited state [23]. Therefore, the signals arise from the $\mathrm{K}$ intermediate, the $\mathrm{L}$ intermediate, and the $\mathrm{N}$ intermediate. 
The 2D-FLCS spectrum generated from a 2D-ILT of the photon histogram [22] calculated at a waiting time of $\Delta t$ $=10 \mu$ s shows three distinct diagonal peaks (Fig. 1f). The cross-peaks between the signals at $\tau^{\prime} \sim 0.08 \mathrm{~ns}$ and $\tau^{\prime} \sim 1.0$ ns indicate rapid exchange between the two states represented by these signals. However, no cross-peaks are observed between the diagonal signal at $\tau^{\prime} \sim 5.0 \mathrm{~ns}$ and the other two states. This is consistent with ensemble experiments which have measured the formation time of the $\mathrm{N}$ intermediate to be on the ms timescale [13, 14]. From these observations we can assign the diagonal peaks at $\tau^{\prime} \sim 0.08 \mathrm{~ns}, \tau^{\prime} \sim 1.0 \mathrm{~ns}$, and $\tau^{\prime} \sim 5.0 \mathrm{~ns}$ to the ground state, the $\mathrm{L}$ intermediate, and the $\mathrm{N}$ intermediates, respectively (Fig. 1c, 1 1f).

To characterize the kinetics of exchange between different intermediates of wt-bR reaction cycle, we measure kinetic traces for waiting times $\Delta t$ ranging from 0.01 to $200 \mathrm{~ms}$ (Figure 2a). In the 2D spectrum, forward and reverse transitions between states $i$ and $j$ appear in the upper and lower quadrant of the spectrum, respectively [20]. The timedependent amplitudes of the spectrum are given by the tcfs $[16,17]$,

$$
C_{i j}(t)=\left\langle S_{i}(t) S_{j}(t+\Delta t)\right\rangle
$$

and

$$
C_{j i}(t)=\left\langle S_{j}(t) S_{i}(t+\Delta t)\right\rangle
$$

where $S_{i}$ and $S_{j}$ are the probabilities the system is in state $i$ and $j$, respectively, measured in our experiment through the unique fluorescence lifetimes $\tau_{i}$ and $\tau_{j}$ of each state. The equivalency of Eq. 1a and Eq. 1b under the condition of microscopic reversibility is given by detailed balance and time-reversal symmetry [21]. Therefore, for reversible transitions, the 2D spectra are symmetric and the dynamics observed through the cross-peaks are identical. Crosspeaks between the $\mathrm{K}$ intermediate and the L intermediate show rapid equilibration of both the forward (Fig. 2b, solid symbols) and reverse reaction (Fig. 2b, open symbols). The $k_{f}$ and $k_{r}$ values for individual transitions are determined from exponential fits of the cross-peak amplitudes. The symmetry of the transitions in the spectra and the equivalency of the kinetic traces suggest microscopically reversible dynamics between the $\mathrm{K}$ and $\mathrm{L}$ intermediate.

Under the condition of microscopic irreversibility, violation of detailed balance and time-reversal symmetry manifests in asymmetric 2D spectra as the cross-correlation functions in Eq. 1a and Eq. 1b are no longer equivalent [21]. At $0.20 \mathrm{~ms}$, a cross-peak emerges between the $\mathrm{L}$ and $\mathrm{N}$ intermediates that is asymmetric (no cross-peak for the reverse transition) (Fig. 2a), indicating a microscopically irreversible step in the reaction cycle. The forward transition occurs on a timescale of $\sim 0.50 \mathrm{~ms}\left(k_{f}=2.0 \times 10^{3} \mathrm{~s}^{-1}\right)$, while the reverse transition occurs on a timescale over $200 \mathrm{~ms}$ $\left(k_{r}=5.0 \mathrm{~s}^{-1}\right)$ (Fig. 2c). This transition represents reprotonation of the Asp96 residue on the cytoplasmic side of the protein ( $\mathrm{M}$ intermediate) and formation of a Grotthuss-like $\mathrm{H}^{+}$wire between the Asp96 residue and the Schiff base of retinal [24-26]. Proton uptake and reprotonation of the retinal Schiff base (formation of $\mathrm{N}$ intermediate) from the cytoplasmic side is thought to be the switch step of the photo-cycle [27-29]. At slightly longer times, we observe asymmetric cross-peaks between the $\mathrm{K}$ intermediate and the $\mathrm{N}$ intermediate (Fig. 2a, d).

The properties of the measured time-correlation functions demonstrate that the reaction cycle of bR is coupled through both microscopically reversible and irreversible transitions. The presence of microscopically irreversible transitions implies that nonequilibrium transitions are inherent to the protein reaction cycle and are required to 


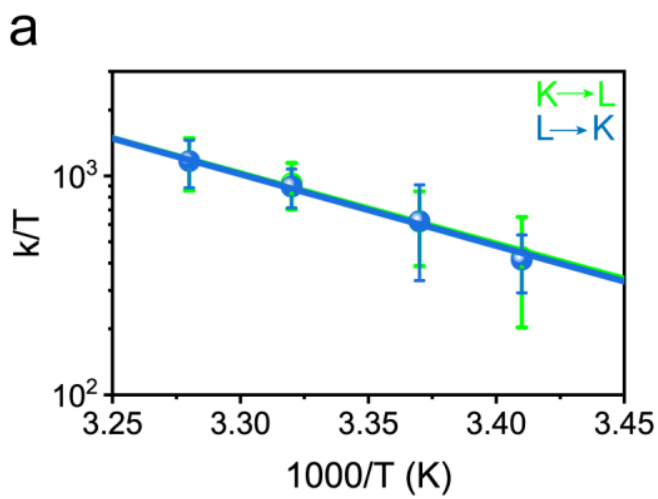

b

C
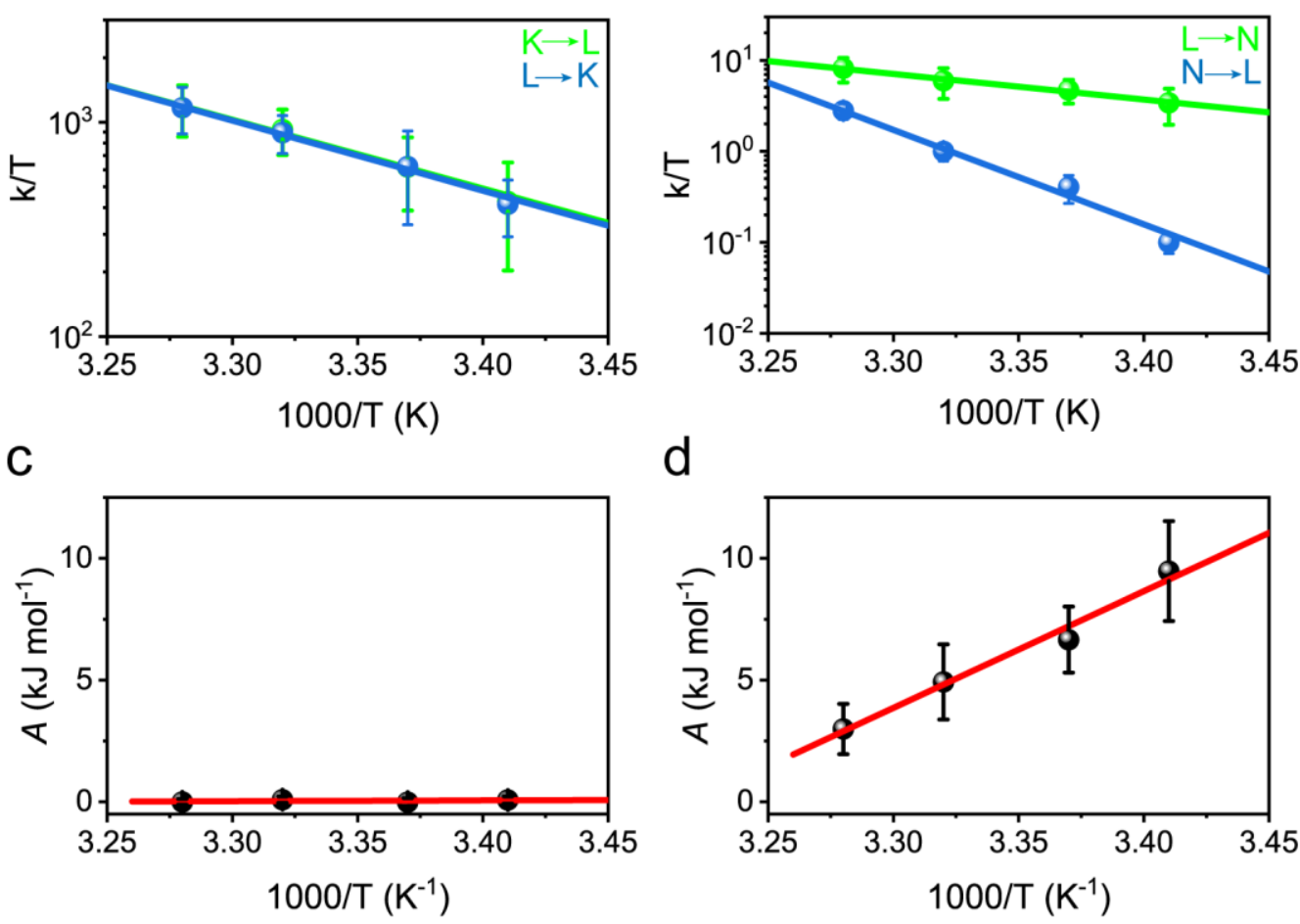

d

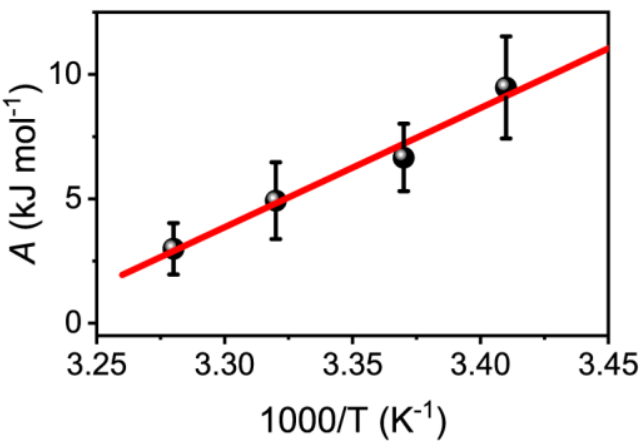

FIG. 3. Thermal stability of the bR reaction cycle. Eyring plots for the K-L (a) and L-N transition rates (b). The $k_{f}$ and $k_{r}$ values are shown in green and blue, respectively. For the reversible K-L transition, the $k_{f}$ and $k_{r}$ values are equal and have the same temperature dependence (a). (c) The affinity $A$ of the K-L transition plotted vs $\mathrm{T}^{-1}$. As the transition is microscopically reversible, the $A$ value is $\sim 0 \mathrm{~kJ} \mathrm{~mol}^{-1}$ at all temperatures measured. For the irreversible L-N transition, the $k_{f}$ and $k_{r}$ values are unequal, indicating a net flux through the transition, and have different temperature dependences (b). (d) The $A$ of the $\mathrm{L}-\mathrm{N}$ transition plotted vs $\mathrm{T}^{-1}$. The microscopically irreversible transition has an $A$ value of $\sim 10 \mathrm{~kJ}$ $\mathrm{mol}^{-1}$ at $291 \mathrm{~K}$ that decreases linearly towards $0 \mathrm{~kJ} \mathrm{~mol}^{-1}$ with increasing temperature. The temperature dependencies of $A$ are fit to the GibbsHelmholtz equation (red line, Eq. 6), highlighting the connection between $A$ and $\Delta G_{r x n}$ for nonequilibrium systems.

maintain stable fluxes through portions of the reaction cycle, even at steady-state. The results also suggest that the stability of the reaction cycle is governed by entropy production associated with irreversible transitions.

Next, we analyze the nonequilibrium thermodynamics of the $b R$ reaction cycle as a function of temperature. In general, the efficiency of a motor protein that relies on microscopic irreversibility should be inversely proportional to temperature, ultimately failing at the point where thermal energy is comparable or larger than the highest activation barrier of the cycle, resulting in zero net flux. Therefore, $k_{f}$ and $k_{r}$ should converge at high temperature for each transition of the reaction cycle.

The $k_{f}$ and $k_{r}$ for an elementary transition are given by Arrhenius expressions,

$$
k_{f}=a e^{\left(-\Delta G_{f}^{\dagger} / k_{B} T\right)}
$$

and,

$$
k_{r}=a e^{\left(-\Delta G_{r}^{\dagger} / k_{B} T\right)}=a e^{\left(-\left[\Delta G_{f}^{\dagger}+\Delta G_{\text {rans }}\right] / k_{B} T\right)}
$$


where $a$ is a pre-exponential factor, $\Delta G_{f}^{\dagger}$ and $\Delta G_{r}^{\dagger}$ are the Gibbs free energy of activation for the forward and reverse transitions, respectively, and $\Delta G_{\text {trans }}$ is the Gibbs free energy of the transition. Assuming the entropic contribution is temperature independent over the range studied, the temperature dependence of $k_{f}$ and $k_{r}$ for a given transition in the cycle will be identical on the condition that $\Delta H_{\text {trans }}=0$ and different when $\Delta H_{\text {trans }} \neq 0$. Eyring plots for the $\mathrm{K}-\mathrm{L}$ transitions and the L-N transitions are shown in Fig. 3a, b. The $k_{f}$ and $k_{r}$ for the K-L transitions show identical temperature dependence (Fig. 3a), consistent with the observed microscopic reversibility of the transition. In contrast, the $k_{f}$ and $k_{r}$ for the L-N transitions (Fig. 3b) converge at high temperature, suggesting that the irreversibility of the transition needed to maintain a net forward flux through the cycle diminishes with increasing temperature.

The affinity $A$ of a nonequilibrium process quantifies the thermodynamic driving force. de Donder demonstrated that $A$ is related to $\Delta G$ for a chemical reaction[30].

$$
A=-\Delta G_{\text {trans }}
$$

Using Eq. 2a-2b, we can rewrite $A$ for a given transition in terms of $k_{f}$ and $k_{r}$.

$$
A=\ln \left(\frac{k_{f}}{k_{r}}\right) \cdot k_{B} T
$$

Plots of $A$ for the K-L and L-N transitions are shown in Fig. 3c, d. The $A$ for the K-L transition, and by extension the $\Delta G_{\text {trans }}$, is $0 \mathrm{~kJ} \mathrm{~mol}^{-1}$ at all temperatures (Fig. 3c). This is consistent with the two states being coupled through reversible dynamics. In contrast, $A$ for the $\mathrm{L}-\mathrm{N}$ transition is $\sim 10 \mathrm{~kJ} \mathrm{~mol}^{-1}$ at $291 \mathrm{~K}$ and decreases rapidly towards $0 \mathrm{~kJ}$ mol $^{-1}$ with increasing temperature (Fig. 3d). According to Eq. 4, the temperature dependence of $A$ is given by the Gibbs-Helmholtz equation.

$$
\frac{\Delta G_{\text {trans }}\left(T_{2}\right)}{T_{2}}-\frac{\Delta G_{\text {trans }}\left(T_{1}\right)}{T_{1}}=\Delta H_{\text {trans }}\left(\frac{1}{T_{2}}-\frac{1}{T_{1}}\right)
$$

Indeed, we find that the experimental data for $A$ as a function of $T$ is adequately fit with Eq. 6, yielding $\Delta H_{\text {trans }} \sim$ $0 \mathrm{~kJ} \mathrm{~mol}^{-1}$ and $\Delta H_{\text {trans }} \sim 30 \mathrm{~kJ} \mathrm{~mol}^{-1}$ for the $\mathrm{K}-\mathrm{L}$ and L-N transitions, respectively (Figure 3c, d).

The thermodynamics of a nonequilibrium process can be quantified by the rate of entropy production rate, $\sigma$, given by [31],

$$
\sigma=\frac{J \cdot A}{T}
$$

where $J$ is the net flux defined for an elementary transition as $\left(k_{f}-k_{r}\right)$. Therefore, experimental measurements of $k_{f}$ and $k_{r}$ are sufficient to determine $\sigma$ of a nonequilibrium chemical process. For K-L and L-N transitions, $\sigma$ is shown as a function of temperature (Fig. 4a). As expected, the K-L transition shows negligible $\sigma$, consistent with the reversible nature of the transition. In contrast, the L-N transitions shows significant $\sigma$, expected for an irreversible transition, that decreases exponentially with increasing temperature. 
a

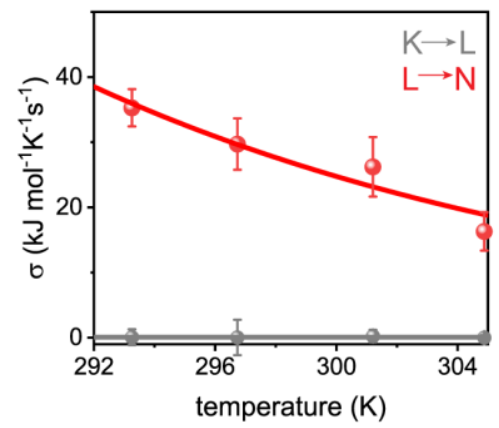

b

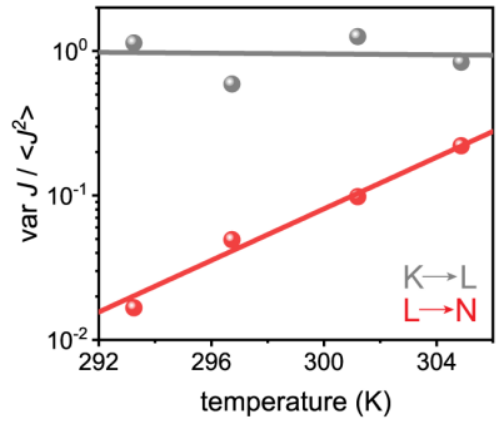

C

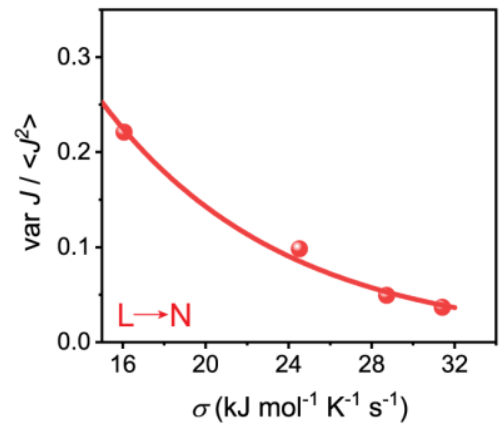

FIG. 4. Entropy production rate and variance of flux. (a) Entropy production for the K-L and L-N transitions computed from Eq. 7. The entropy production rate $\sigma$ associated with the reversible K-L transition is $0 \mathrm{~kJ} \mathrm{~mol}^{-1} \mathrm{~K}^{-1} \mathrm{~s}^{-1}$ at all temperatures measured, while the irreversible $\mathrm{L}-\mathrm{N}$ transition has significant entropy production rate $\left(\sim 40 \mathrm{~kJ} \mathrm{~mol}^{-1} \mathrm{~K}^{-1} \mathrm{~s}^{-1}\right)$ that decreases exponentially with increasing temperature. (b) Plot of varJ/<J $>$ as a function of temperature. For the reversible K-L transition, the variance of flux remains constant with temperature. In contrast, the irreversible L-N transition shows a small variance in flux at $291 \mathrm{~K}$ that exponentially increases with increasing temperature. (c) Plot of varJ/ $\left\langle J^{2}\right\rangle$ as a function of $\sigma$ for the L-N transition. The results demonstrate that the variance of flux through a given transition of the reaction cycle decreases exponentially as $\sigma$ of the transition increases.

Entropy production ensures directionality through a nonequilibrium reaction cycle. For a dynamic process, the probability of observing a given dynamic trajectory compared to its time-reversed counterpart is related to the entropy production rate associated with the process through the fluctuation theorem [32],

$$
\frac{P(\sigma)}{P(-\sigma)}=e^{\tau \sigma / k_{B} T}
$$

where $P(\sigma)$ is the probability of observing a given microscopic trajectory, and $P(-\sigma)$ is the probability of observing the time-reversed microscopic trajectory, over a trajectory of time $\tau$. For a nonequilibrium biochemical reaction cycle, $P(\sigma)$ represents the forward flux through the cycle and $P(-\sigma)$ the reverse flux. If the forward flux through the cycle is accompanied by a large $\sigma$, then it is heavily favored over the reverse flux.

To characterize the stability of a molecular motor we want to determine the variance of flux, or noise, associated with the reaction cycle. Here, we use maximum caliber method to estimate the variance of flux through the experimentally observed transitions [33-36]. In this method, the most probable distribution of pathways dynamically coupling two states is determined by maximizing the path Shannon entropy subject to constraints from experimentally measured fluxes. From the so-called dynamical partition function determined from maximum caliber, properties such as the variance of flux for a transition can be computed. Applying this method to biochemical reaction cycles, Dill and coworkers derived expressions for the mean and variance of flux for a given transition of the cycle [37],

$$
\langle J\rangle=\frac{2 N}{s} \varepsilon
$$

and,

$$
\operatorname{var} J=\frac{2 N}{s^{2}}\left(k_{0}-2 \varepsilon^{2}\right)
$$


where $N$ is the number of discrete steps in a trajectory, $s$ is the number of intermediates in the reaction cycle, $k_{0}$ is the inherent rate of a transition common for the forward and reverse transition, and $\varepsilon$ is the deviation from the inherent rate constant due to the violation of detailed balance. The experimentally measured $k_{f}$ and $k_{r}$ are easily transformed into $k_{0}$ and $\varepsilon$ values, and $N$ can be determined from Eq. 9a using the measured mean fluxes.

Fig. 4b shows varJ $\left\langle J^{2}\right\rangle$, computed from Eq. 9b, as a function of temperature. For the microscopically reversible $\mathrm{K}-\mathrm{L}$ transition, the variance is both maximized and constant as a function of temperature (Fig. 4b). In contrast, for the irreversible transition of the reaction cycle, we observe the variance of flux increases exponentially with increasing temperature. The transition from the $\mathrm{L}$ intermediate to the $\mathrm{N}$ intermediate covers the switch step of the reaction cycle of wt-bR. The failure of wt-bR (nondirectional $\mathrm{H}^{+}$pumping) at high temperature can therefore be understood as resulting from the exponentially increasing variance of flux through the reaction cycle. Fig. 4c shows a plot of $\operatorname{var} J /\left\langle J^{2}\right\rangle$ as a function of $\sigma$. The data shows that the variance of flux through the reaction cycle, and therefore the probability of observing reverse transitions through the cycle, diminish exponentially with $\sigma$, consistent with Eq. 8. This is likely a general mechanism for thermal instability for biological machineries that rely on microscopic irreversibility.

The mechanism by which biological machinery overcomes randomizing thermal forces to achieve directional action are not comprehensively understood, with key questions regarding microscopic reversibility and the applicability of equilibrium dynamics still remaining. The results presented here demonstrate that microscopic irreversibility is the fundamental operating principle of directional $\mathrm{H}^{+}$transport by bacteriorhodopsin. Using multidimensional single-molecule spectroscopy, we demonstrate that the reaction cycle is comprised of both reversible and irreversible conformational transitions, with the entropy production associated with irreversible transitions ensuring directionality of the cycle (and hence the directionality of $\mathrm{H}^{+}$transport). Therefore, equilibrium notions of detailed balance and time-reversal symmetry proposed for chemically driven motors are not applicable to the function of optically driven motors. Similarly, the thermal destabilization of the reaction cycle of bacteriorhodopsin is likely unique to motors that operate through a power-stroke mechanism in which the nonequilibrium nature of the cycle is inherent to select conformational transitions of the protein.

\section{ACKNOWLEDGEMENTS}

We thank F. Amblard, A. Shakya, and G. Paneru for productive discussions. We also thank the Korean taxpayers for supporting this work through the Korean Institute for Basic Science, Project Code IBS-R020-D1.

\section{REFERENCES}

[1] R.D. Vale, R.A. Milligan, Science, 288 (2000) 88-95.

[2] S. Leibler, D.A. Huse, J. Cell Biol., 121 (1993) 1357-1368.

[3] W. Hwang, M. Karplus, Proc. Natl. Acad. Sci. U.S.A., 116 (2019) 19777-19785.

[4] Y. Taniguchi, M. Nishiyama, Y. Ishii, T. Yanagida, Nat. Chem. Biol., 1 (2005) 342-347.

[5] J.A. Wagoner, K.A. Dill, Proc. Natl. Acad. Sci. U.S.A., 116 (2019) 5902-5907.

[6] R.D. Astumian, Nat. Nanotechnol., 7 (2012) 684-688.

[7] R.D. Astumian, Science, 276 (1997) 917-922. 
[8] F. Julicher, A. Ajdari, J. Prost, Rev. Mod. Phys., 69 (1997) 1269-1281.

[9] S.M. Simon, C.S. Peskin, G.F. Oster, Proc. Natl. Acad. Sci. U.S.A., 89 (1992) 3770-3774.

[10] J. Howard, Curr. Biol., 16 (2006) 517-519.

[11] R.D. Astumian, Faraday Discuss., 195 (2016) 583-597.

[12] H. Luecke, B. Schobert, H.T. Richter, J.P. Cartailler, J.K. Lanyi, J. Mol. Biol., 291 (1999) 899-911.

[13] J.K. Lanyi, Annu. Rev. Physiol., 66 (2004) 665-688.

[14] O.P. Ernst, D.T. Lodowski, M. Elstner, P. Hegemann, L.S. Brown, H. Kandori, Chem. Rev., 114 (2014) 126163.

[15] C. Maes, K. Netocny, J. Stat. Phys., 110 (2003) 269-310.

[16] K. Ishii, T. Tahara, J. Phys. Chem. B, 117 (2013) 11414-11422.

[17] K. Ishii, T. Tahara, J. Phys. Chem. B, 117 (2013) 11423-11432.

[18] T. Otosu, K. Ishii, T. Tahara, Nat. Commun., 6 (2015) 1-9.

[19] T. Kondo, J.B. Gordon, A. Pinnola, L. Dall'Osto, R. Bassi, G.S. Schlau-Cohen, Proc. Natl. Acad. Sci. U.S.A., 116 (2019) 11247-11252.

[20] W.P. Aue, E. Bartholdi, R.R. Ernst, J. Chem. Phys., 64 (1976) 2229-2246.

[21] I.Z. Steinberg, Biophys. J., 50 (1986) 171-179.

[22] S. Talele, J.T. King, BioRxiv, ms 2021/425066 (2021).

[23] P. Nogly, T. Weinert, D. James, S. Carbajo, D. Ozerov, A. Furrer, D. Gashi, V. Borin, P. Skopintsev, K. Jaeger, K. Nass, P. Bath, R. Bosman, J. Koglin, M. Seaberg, T. Lane, D. Kekilli, S. Brunle, T. Tanaka, W.T. Wu, C. Milne, T. White, A. Barty, U. Weierstall, V. Panneels, E. Nango, S. Iwata, M. Hunter, I. Schapiro, G. Schertler, R. Neutze, J. Standfuss, Science, 361 (2018).

[24] F. Garczarek, K. Gerwert, Nature, 439 (2006) 109-112.

[25] E. Freier, S. Wolf, K. Gerwert, Proc. Natl. Acad. Sci. U.S.A., 108 (2011) 11435-11439.

[26] T. Weinert, P. Skopintsev, D. James, F. Dworkowski, E. Panepucci, D. Kekilli, A. Furrer, S. Brunle, S. Mous, D. Ozerov, P. Nogly, M.T. Wang, J. Standfuss, Science, 365 (2019) 61-65.

[27] J.K. Lanyi, Biochim. Biophys. Acta, 1183 (1993) 241-261.

[28] M. Kataoka, H. Kamikubo, F. Tokunaga, L.S. Brown, Y. Yamazaki, A. Maeda, M. Sheves, R. Needleman, J.K. Lanyi, J. Mol. Biol., 243 (1994) 621-638.

[29] G. Varo, J.K. Lanyi, Biochemistry, 30 (1991) 5008-5015.

[30] T. de Donder, P.V. Rysselberghe, Thermodynamic Theory of Affinity, Stanford University Press, California, 1936.

[31] S.R. de Groot, P. Mazur, Non-equilibrium thermodynamics, Dover, New York, 1962.

[32] G.E. Crooks, Phys. Rev. E, 60 (1999) 2721-2726.

[33] E.T. Jaynes, Phys. Rev., 106 (1957) 620-630.

[34] E.T. Jaynes, Phys. Rev., 108 (1957) 171-190.

[35] R.C. Dewar, J. Phys. A; Math. Gen., 38 (2005) L371-L381.

[36] S. Presse, K. Ghosh, J. Lee, K.A. Dill, Rev. Mod. Phys., 85 (2013) 1115-1141.

[37] S. Presse, K. Ghosh, R. Phillips, K.A. Dill, Phys. Rev. E, 82 (2010). 


\title{
Supplemental Information
}

\section{Stability of a Nonequilibrium Biochemical Cycle Revealed}

\section{by Single-Molecule Spectroscopy}

\author{
Saurabh Talele ${ }^{1,2}$ and John T. King ${ }^{1 *}$ \\ ${ }^{1}$ Center for Soft and Living Matter, Institute for Basic Science, Ulsan 44919, Republic of Korea \\ ${ }^{2}$ Department of Biomedical Engineering, Ulsan National Institute of Science and Technology, Ulsan 44919, \\ Republic of Korea \\ Email: jtking@unist.ac.kr
}

Materials. Purified and lyophilized wt-bR and bR-D96N extracted from Halobacterium salinarum were purchased from Halotek biomaterials (Halotek, Germany). The sample was loaded into UV-treated 8-well chambers (Lab-Tek, ThermoFisher, USA) for single-molecule experiments. Single-molecule fluorescence experiments were carried out on a commercial Leica SP8x microscope (Leica Microsystems GmbH, Germany). Pulsed excitation light at 532 nm was provided by a Fianium Supercontinuum Laser operating at $40 \mathrm{MHz}$ with an average power of $\sim 6 \mu \mathrm{W}$ at the backfocal-plane. Excitation and collection of fluorescence emission was carried out through a 100X oil-immersion objective. Fluorescence signal was passed through a band-pass filter (Semrock, USA) and was detected by photon avalanche photodiodes (PicoQuant, Germany) equipped with a picoHarp300 (PicoQuant, Germany) time-correlated single-photon-counting (TCSPC) system. Data processing and analysis was performed using in-house MatLab code.

Sample Preparation. A $1 \mu \mathrm{M}$ solution of membrane-bound bacteriorhodopsin (bR) was prepared in phosphate buffer (100 mM ionic strength, $\mathrm{pH}=6$ ). This solution was vortexed and sonicated for 5 minutes in cold water to disperse the purple membrane. The dispersed suspension was centrifuged for 5 minutes at $10 \mathrm{~K} \mathrm{rpm}$. The supernatant was extracted, and the sonication/centrifuge procedure was repeated 3 times. The third-degree supernatant ( $1 \mathrm{nM} \mathrm{bR})$ was used for experiments. An 8-well chamber (LabTek, Australia) was UV treated for $>8$ hrs prior to use. The bR solution was 
a

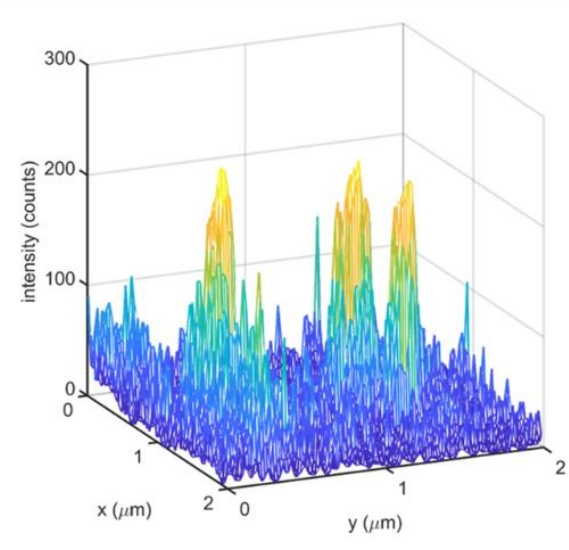

C

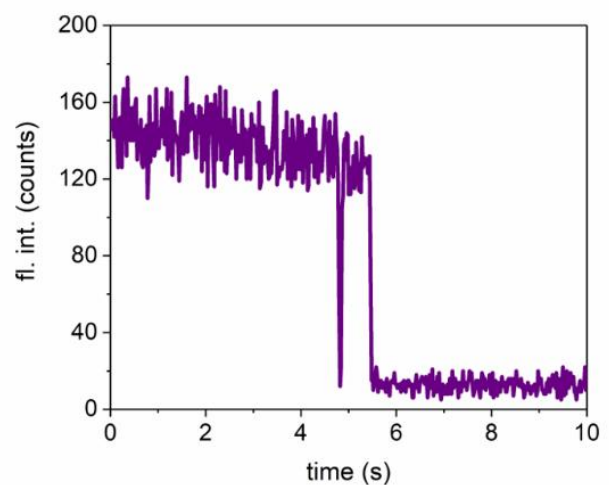

b

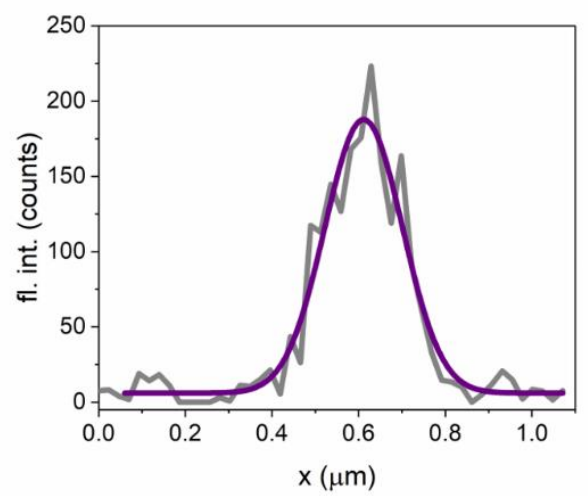

d

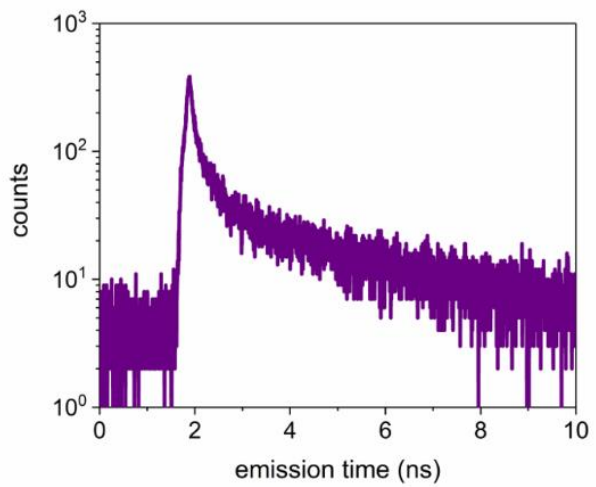

FIG. S1. Single-molecule imaging and photo-physics. (a) Confocal image of single Bacteriorhodopsin proteins immobilized on a glass surface. (b) Diffraction-limited cross-section of fluorescence signal measured in confocal image. (c) Single-molecule fluorescence trace showing singlestep photo-physics. (d) Fluorescence lifetime histogram from single wt-bR protein showing multicomponent relaxation.

then added to the chamber and kept at ambient light for 10-15 minutes. Membrane bound bR adsorb to the glass surface and are immobilized.

SM-2D-FLCS. Single-molecule dispersion and immobilization of bR was confirmed by confocal microscopy and fluorescence time traces (Fig. S1a-c). Emission from bR showed diffraction limited detection spots (Fig. S1a, b) and single-step photo-physics (Fig. S1c) expected for single molecules. Excitation at $532 \mathrm{~nm}$ overlaps with the absorption spectrum of the retinal chromophore (in ground state, $\mathrm{K}, \mathrm{L}$, and $\mathrm{N}$ intermediate states) embedded in the protein scaffold. The excitation laser both initiates the catalytic cycle and probes transient intermediates formed during the reaction cycle. Fluorescence relaxation from a single bR protein shows multi-component relaxation (Fig. S1d). The emission delay times extracted from a real-time photon stream (Fig. S2a) can be binned into a 1D histogram which are used to estimate a relaxation spectrum through an inverse Laplace transformation (Fig. S2b). For a two-state system where 
a

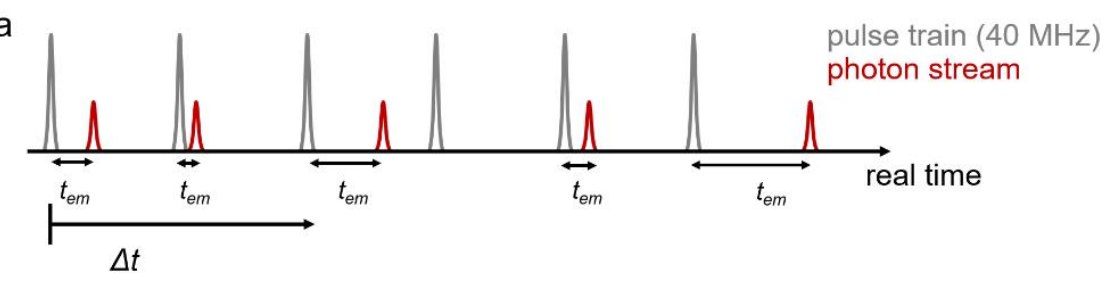

b

1D photon histogram
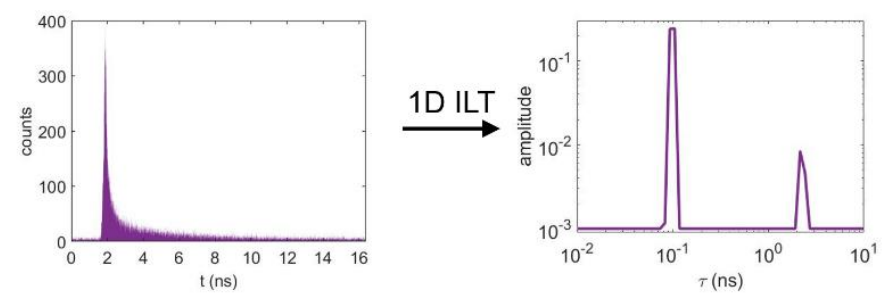

C

2D photon histogram
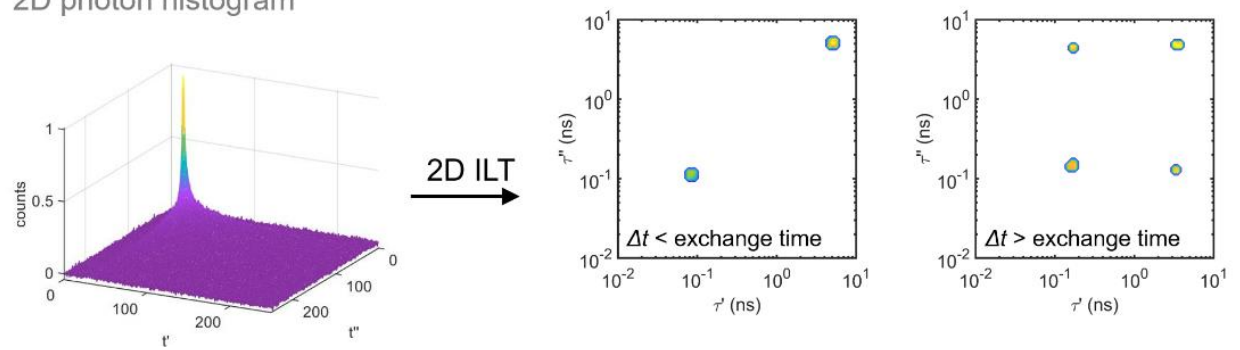

FIG. S2. Experimental procedure for measuring sm-2D-FLCS data. (a) Excitation pulse train and photon stream measured with resolved photon emission time $t_{e m}$ (standard TCSPC measurement in Time-Tagged-Time-Resolved mode). (b) 1D histogram of photon emission time and the fluorescence lifetime spectrum computed by a 1D ILT for a simple two state system. (c) 2D histogram of photon emission measured at a set waiting time $\Delta t$ and 2D-FLCS spectra computed by a 2D ILT. At early waiting times ( $\Delta t<$ exchange time) peaks are only measured along the diagonal, indicating species that remained in their initial state during the waiting time. At later times ( $\Delta t>$ exchange time), cross-peaks arise as species that were in an initial state exchange to the other state during the waiting time.

each state has a distinct fluorescence lifetime the 1D fluorescence lifetime spectrum will show a peak at the relaxation rate of each state. 2D-FLCS spectra are computed by generating a 2D photon histogram of photon pairs separated by a systematic waiting time $\Delta t$ (Fig. S2a). A 2D inverse Laplace transformation of the histogram gives a 2D-FLCS spectrum for a given $\Delta t$ (Fig. S2c). For a two-state system that undergoes chemical exchange at a well-defined timescale, the 2D-FLCS spectrum will contain diagonal peaks corresponding to species that did not exchange within $\Delta t$ and cross-peaks corresponding to species that did exchange within $\Delta t$ (Fig. S2c). Monitoring the kinetics of the cross-peaks provides direct access to the forward and reverse exchange times. 
Inverse Laplace Transformations. 1D and 2D inverse Laplace transformation (ILT) of the measured photon streams were performed through in-house MATLAB code described elsewhere [1]. From a photon stream collected from a single-molecule a 2D histogram is generated where each pixel denotes the number of coincidence events within the time trace where a photon with emission delay of $t_{2}$ is observed after $\Delta t$ from observation of a photon with delay time $t_{l}$. This represents the $2 \mathrm{D}$ emission delay correlation map, $X\left(t_{l}, t_{2}\right)$, which can be expressed as,

$$
X\left(t_{1}, t_{2}\right)=\iint k_{1}\left(t_{1}, \tau_{1}\right) S\left(\tau_{1}, \tau_{2}\right) k_{2}\left(t_{2}, \tau_{2}\right) d \tau_{1} d \tau_{2}
$$

where $S\left(\tau_{1}, \tau_{2}\right)$ is the $2 \mathrm{D}$ fluorescence correlation spectrum, $\tau$ 's represents fluorescence lifetimes, and $k_{1}$ and $k_{2}$ are emission delay kernels. $S\left(\tau_{1}, \tau_{2}\right)$ is estimated by computing a 2D ILT of $X\left(t_{1}, t_{2}\right)$ at various $\Delta t$. This effectively decomposes the total correlation function into the autocorrelation function (diagonal elements) and cross-correlation function (off-diagonal elements) of the constituent components in the single-molecule fluorescence time trace. As ILTs are numerically unstable and cannot be solved analytically, they instead must be estimated. Tahara and coworkers [2] used Maximum Entropy Method (MEM) [3] to estimate the 2D-FLCS spectrum, though this approach can be computationally costly. An alternative approach, based on recent advances in 2D NMR spectroscopy [4, 5], uses Tikhonov regularization [6] instead of MEM and exploits the kernel structure for efficient data compression via singlevalue decomposition, thus greatly reducing the computational costs without sacrificing the quality of fit of lifetime resolution [1]. We adapted this technique for the computation of sm-2D-FLCS spectra. For each $\Delta t$, the spectrum was computed using the photons within a time window for which the denoted $\Delta t$ was the upper limit (see Photon Windows Section below).

Photon Windows. sm-2D-FCLS spectra are computed using photons measured within a time window which is denoted by a single waiting time $\Delta t$. The time windows used for each waiting time are given below:

\begin{tabular}{ll} 
Time Window $(s)$ & Denoted $\Delta t(s)$ \\
\hline $1 \times 10^{-6}-1 \times 10^{-5}$ & $1 \times 10^{-5}$ \\
$1 \times 10^{-5}-5 \times 10^{-5}$ & $5 \times 10^{-5}$ \\
$5 \times 10^{-5}-2 \times 10^{-4}$ & $2 \times 10^{-4}$ \\
$2 \times 10^{-4}-5 \times 10^{-4}$ & $5 \times 10^{-4}$ \\
$5 \times 10^{-4}-1 \times 10^{-3}$ & $1 \times 10^{-3}$ \\
$1 \times 10^{-3}-1 \times 10^{-2}$ & $1 \times 10^{-2}$ \\
$1 \times 10^{-2}-5 \times 10^{-2}$ & $5 \times 10^{-2}$ \\
$5 \times 10^{-2}-2 \times 10^{-1}$ & $2 \times 10^{-1}$
\end{tabular}


bioRxiv preprint doi: https://doi.org/10.1101/2021.05.24.445545; this version posted May 25, 2021. The copyright holder for this preprint (which

was not certified by peer review) is the author/funder, who has granted bioRxiv a license to display the preprint in perpetuity. It is made available under aCC-BY-NC-ND 4.0 International license.
$2 \times 10^{-1}-5 \times 10^{-1}$
$5 \times 10^{-1}$
$5 \times 10^{-1}-1 \times 10^{0}$
$1 \times 10^{0}$

\section{REFERENCES}

[1] S. Talele, J.T. King, BioRxiv, ms 2021/425066 (2021).

[2] K. Ishii, T. Tahara, J. Phys. Chem. B, 117 (2013) 11414-11422.

[3] J. Skilling, R.K. Bryan, Mon. Not. R. Astron. Soc., 211 (1984) 111-124.

[4] L. Venkataramanan, Y.Q. Song, M.D. Hurlimann, IEEE Trans. Signal Process., 50 (2002) 1017-1026.

[5] Y.Q. Song, L. Venkataramanan, M.D. Hurlimann, M. Flaum, P. Frulla, C. Straley, J. Magn. Reson., 154 (2002) 261-268.

[6] A.N. Tikhonov, Dokl. Akad. Nauk SSSR, 151 (1963) 1035-1038. 


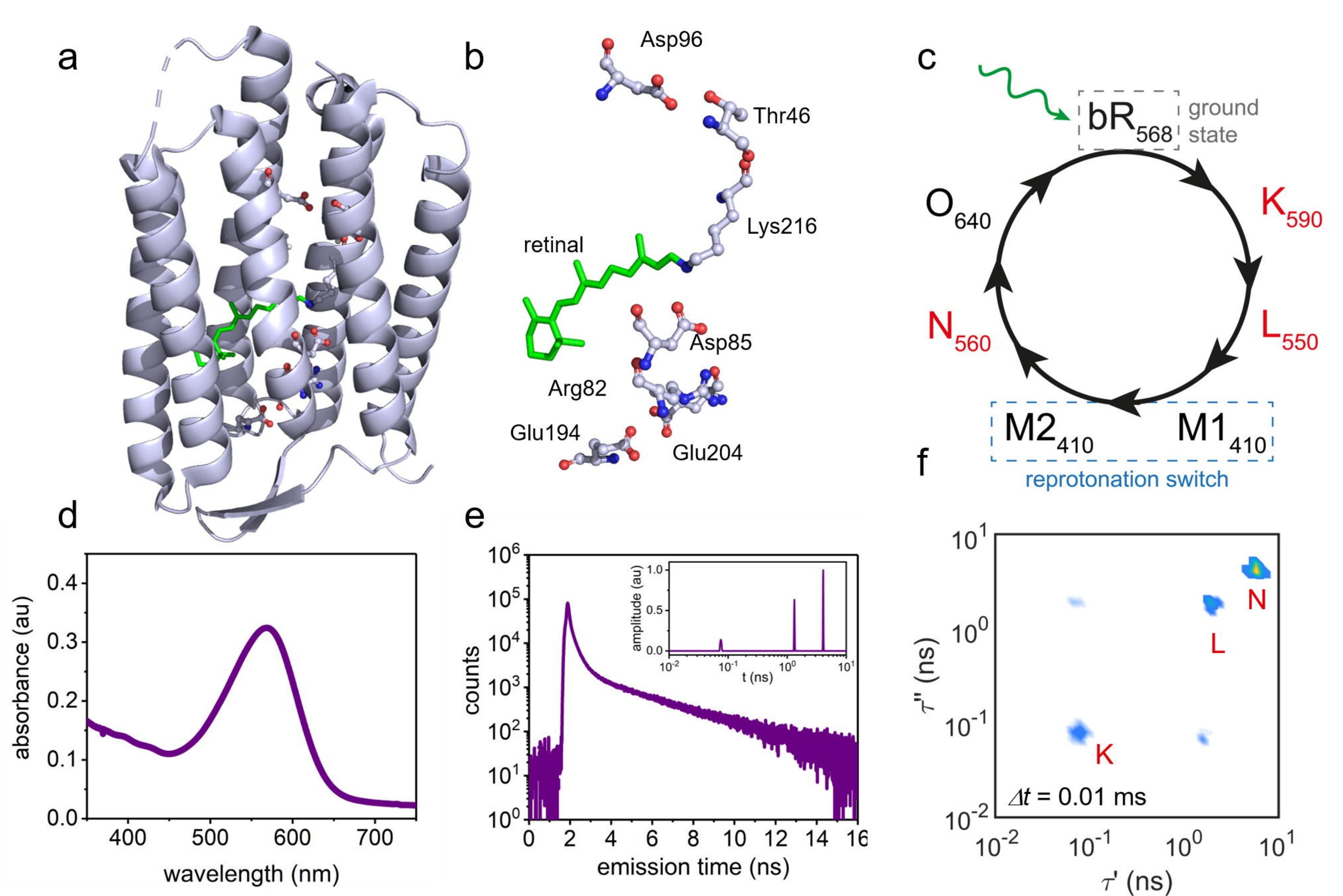




\section{a}
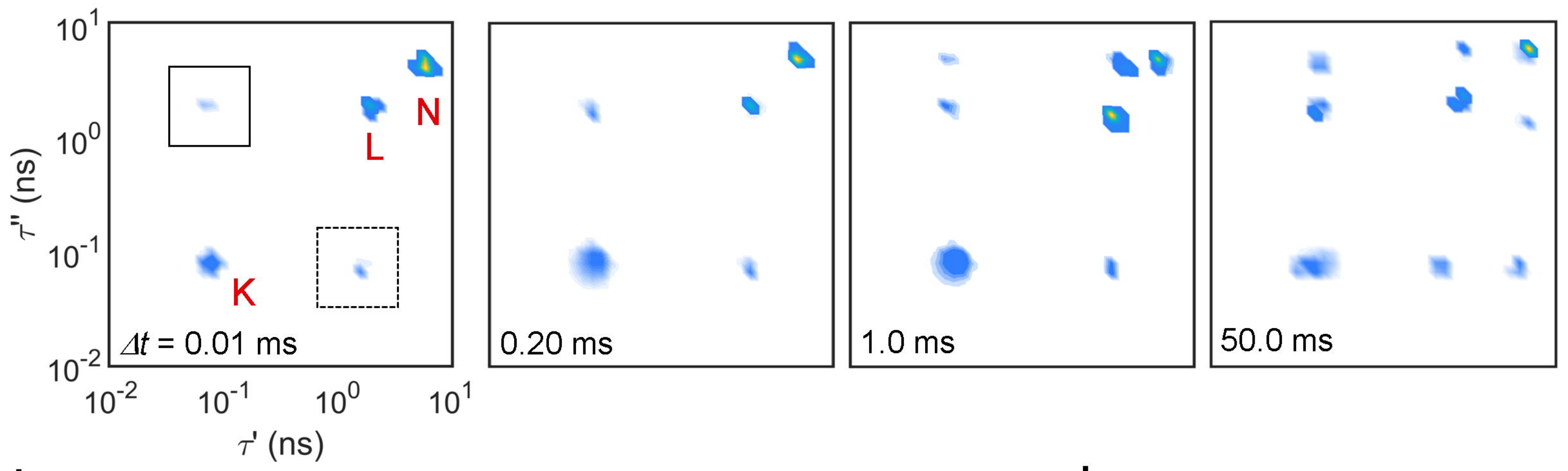

b

C

d
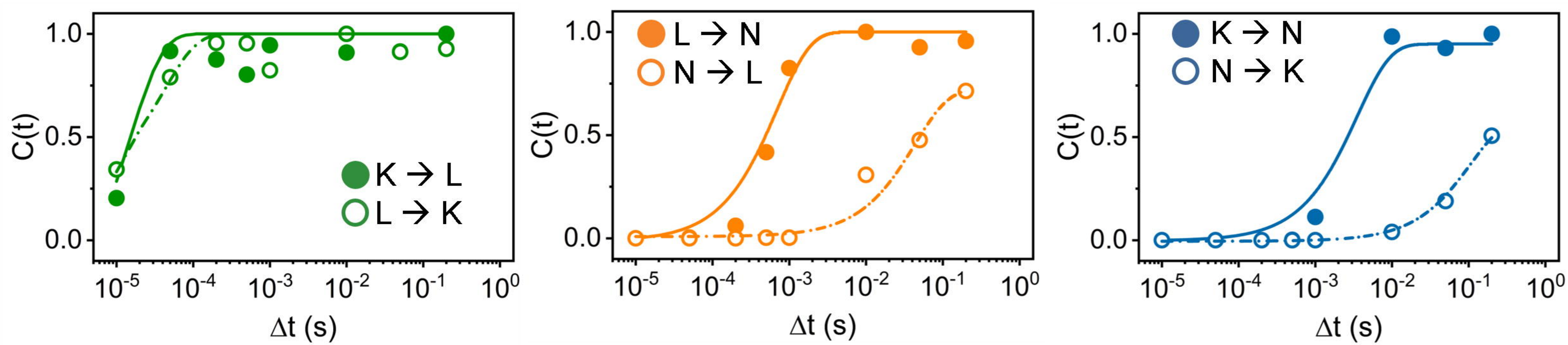
a

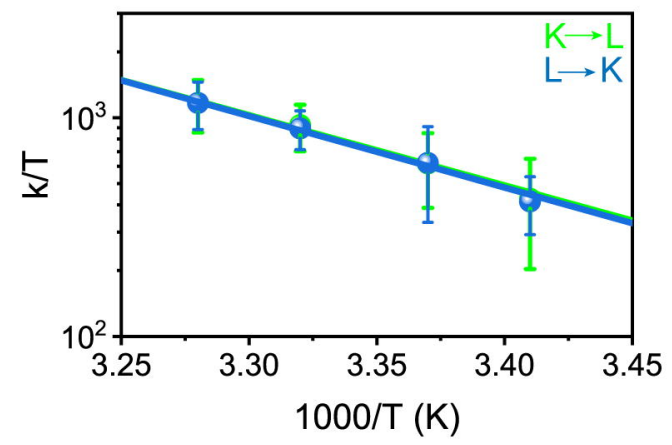

C

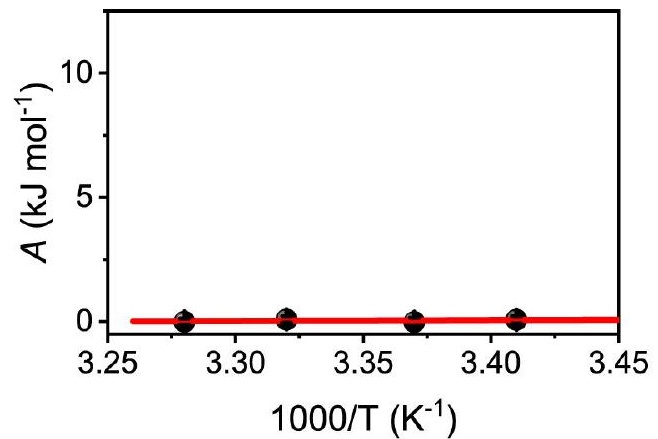

b
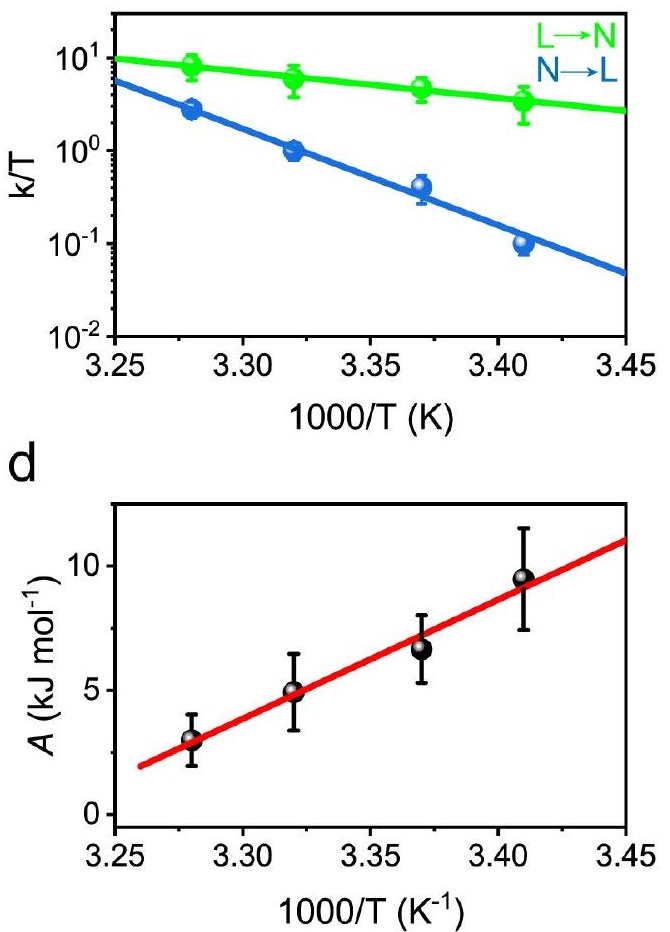


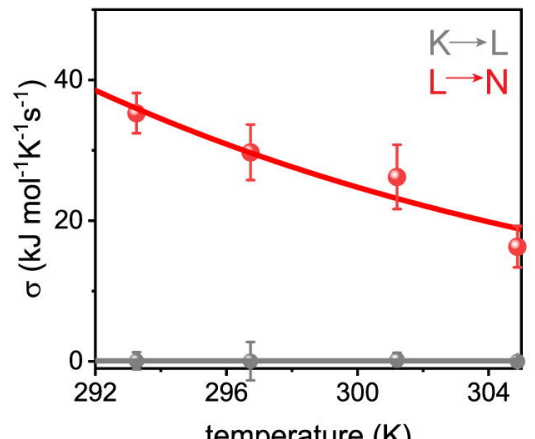

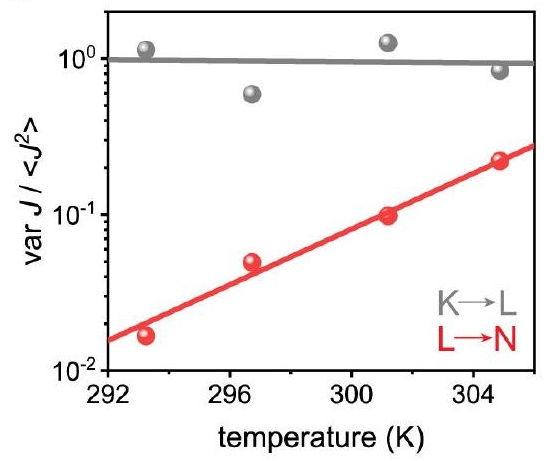

C

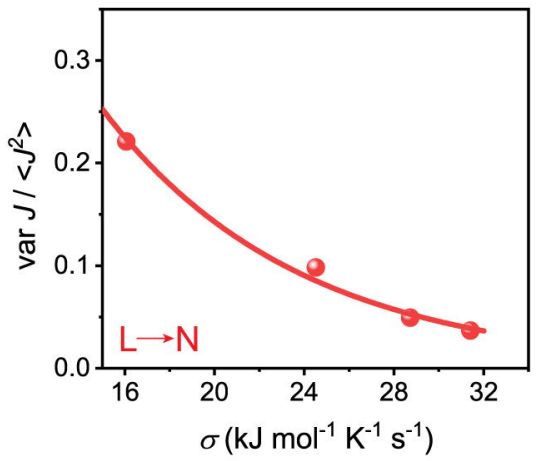

